\title{
Un futuro posible para el psicoanálisis'
}

\author{
Miguel Ángel González Torres² \\ Universidad del País Vasco
}

La pérdida de Horst Kächele nos empuja a una reflexión sobre su persona y su obra, centrada toda ella en el examen de los problemas del Psicoanálisis y el modo de resolverlos. Su concepción positivista de nuestra disciplina le llevó a practicar, enseñar e investigar de un modo a la vez abierto y riguroso. Abierto a opiniones diferentes, a críticas y cuestionamientos. Riguroso en su concepción de la investigación empírica como eje del futuro del psicoanálisis. A partir de una interpretación personal de sus propuestas, se dibujan aquí unas líneas maestras de lo que podría ser un psicoanálisis del futuro, que proteja de la irrelevancia cultural y científica a nuestro campo y posibilite un crecimiento fértil. Vincular el Psicoanálisis a la Universidad, revisar en profundidad los programas de formación y optar por un apoyo decidido a la investigación se sitúan como elementos centrales en ese posible futuro.

Palabras clave: Psicoanálisis, Investigación, Formación, Obra y significación de Horst Kächele

The loss of Horst Kächele prompts us to reflect on his person and his work, all centered on examining the problems of Psychoanalysis and how to solve them. His positivist conception of our discipline led him to practice, teach and investigate in an open and rigorous way. Open to different opinions, criticisms and questions. Rigorous in his conception of empirical research as the axis of the future of psychoanalysis. Starting from a personal interpretation of his proposals, some master lines are drawn here of what a psychoanalysis of the future could be, which protects our field from cultural and scientific irrelevance and enables fertile growth. Linking Psychoanalysis to the University, reviewing the training programs in depth and opting for decisive support for research are central elements in this possible future.

Key Words: Psychoanalysis, Research, Training, Significance and Work of Horst Kächele

\section{English Title: A possible future for psychoanalysis}

\section{Cita bibliográfica / Reference citation:}

González Torres, M.A. (2020). Un futuro posible para el psicoanálisis. Clínica e Investigación Relacional, 14 (2): 349-364. [ISSN 1988-2939] [Recuperado de www.ceir.info ] DOI: $10.21110 / 19882939.2020 .140205$

\footnotetext{
${ }^{1}$ Trabajo escrito en memoria de Horst Kächele, que falleció este mismo año. Véase también la reseña In Memoriam que CelR le dedica en este mismo número.

2 Departamento de Neurociencias. Universidad del País Vasco. Servicio de Psiquiatría. Hospital Universitario de Basurto. Bilbao. Centro Psicoanalítico de Madrid. Contacto: miguelangel.gonzaleztorres@osakidetza.eus
} 
El reciente fallecimiento de Horst Kächele supone un acontecimiento doloroso en el mundo psicoanalítico. Tanto quienes gozaron de su amistad como quienes asistimos a sus presentaciones y seminarios y admiramos sus textos sentimos su pérdida y lamentamos su ausencia definitiva. El conjunto de su obra nos interpela y de algún modo nos obliga a reflexionar sobre las cuestiones que abordó en su larga y productiva carrera clínica e investigadora. Los pensadores de la talla de Kächele nos entregan al partir una antorcha que deberíamos recoger.

Abordó buena parte de las cuestiones fundamentales del Psicoanálisis. Creyó firmemente en las bondades de nuestra técnica y en la excelencia de sus resultados. Y sobre todo creyó en la necesidad de retirar al psicoanálisis el ropaje religioso que todavía para algunos conserva. Se mostró siempre crítico con la asunción de dogmas de fe y abogó por una actitud abiertamente cuestionadora, comenzando por plantear dudas respecto a cuestiones consideradas "sagradas" en nuestra disciplina como el uso del diván, las diferencias entre psicoterapia y psicoanálisis, el lugar de la interpretación transferencial como elemento único de la acción terapéutica o la relevancia de la contratransferencia y señaló la obligación imperiosa de buscar evidencias de los resultados de los tratamientos.

Podríamos decir que Kächele proponía, y mostraba, una actitud valiente, abierta al debate y enemiga de puertas cerradas y camarillas protectoras. Una actitud que podemos considerar plenamente científica. En la mejor tradición del primer psicoanálisis y de ese Freud escéptico, pesimisita y curioso que Stoller (2009; p 214) reclamaba como suyo.

Una manera de recoger el testigo de Kächele es reflexionar sobre el psicoanálisis del futuro. Aquel al que a algunos nos gustaría llegar. Teniendo a su obra como compañía y orientación; pensar, o más bien soñar, el porvenir de esta ciencia llamada psicoanálisis. Claramente se trata de un sueño obligadamente compartido. Son muchos los analistas que se sienten influidos por las propuestas y las reflexiones de este autor $y$, obviamente, cualquier progreso futuro de nuestra disciplina se alcanzará gracias a la colaboración y el entusiasmo de muchos.

También en este sentido Kächele nos marca un camino pues una parte importante de su obra ha sido fruto de un esfuerzo plural. En nuestro campo los artículos con un solo autor son mayoría abrumadora. No es el caso de Kächele que reflexionaba casi siempre en compañía. Especialmente junto a dos autores que podemos señalar. Helmut Thöma es el primero de ellos. Ambos redactaron el influyente texto "Teoría y Práctica del Psicoanálisis" (dos tomos, 1985 y 1989, con una segunda edición pendiente de publicar en castellano). Ambos autores compartían una estrecha relación que dio frutos en lo personal y en lo científico. El espíritu abierto y cuestionador fue propio de ambos y caracterizó toda su 
importante obra conjunta. El otro coautor principal de Kächele es el norteamericano Joseph Schachter, con quien escribió un libro fundamental: "Nodal Points: Critical Issues in Contemporary Psychoanalytic Therapy" (Schachter \& Kächele 2017) que nos sitúa frente a las grandes controversias actuales forzando al lector a la reflexión más activa.

¿A dónde podrían llevarnos las propuestas de Kächele y sus colegas?. Intentaré esbozar aquí algunas líneas que creo fundamentales. Obviamente no puedo afirmar en absoluto que Kächele estaría de acuerdo con lo que planteo, sino tan solo que mi lectura de algunas de sus obras, junto a las de otros autores, me sirven de orientación para diseñar un particular panorama futuro, desde luego para mí deseable. Quienes conocen la obra de Kächele podrán valorar además si este esquema refleja en alguna medida su visión personal.

\section{¿Qué es psicoanálisis?}

Podemos considerar "Psicoanálisis" al conjunto de técnicas basadas en la teoría psicoanalítica que se emplean como herramienta terapéutica en una amplia variedad de patologías y problemas clínicos. Estas técnicas abarcan desde las intervenciones de apoyo psicoanalíticamente orientadas hasta el psicoanálisis en su formato tradicional, pasando por una amplia variedad de psicoterapias psicoanalíticas, de introspección, exploratorias...tanto breves como de larga duración. Los diferentes problemas clínicos deben ser atendidos desde la técnica que haya demostrado mayor eficacia. La idea de que la intervención psicoanalítica tradicional (diván y varias sesiones semanales) es la intervención idónea para una gran mayoría de problemas es errónea. La idea de que las demás intervenciones psicoanalíticas deben utilizarse solo cuando el formato tradicional no es viable, también.

La teoría psicoanalítica se fundamenta en tres postulados nucleares (Solms, 2020): A) El bebé humano nace con una serie de necesidades innatas. B) El desarrollo psíquico consiste en el aprendizaje para cubrir esas necesidades en el mundo real. C) La mayor parte de esos procesos dirigidos a cubrir las necesidades innatas ocurren de forma inconsciente. Los tres postulados subyacen a todos y cada uno de los modelos teóricos psicoanalíticos, que introducen múltiples matices diferentes sobre estos elementos centrales. Los tres postulados encajan perfectamente a día de hoy con las evidencias disponibles desde el propio psicoanálisis, las ciencias cognitivas o la neurobiología, de tal modo que sigue estando plenamente vigente la afirmación del premio Nobel Eric Kandel (1999) cuando 
señaló que "el psicoanálisis todavía representa la visión más coherente e intelectualmente satisfactoria de la mente".

Freud (1933) se muestra ambivalente a la hora de considerar lo terapéutico como la mayor contribución del Psicoanálisis. Cree firmemente en su capacidad para revelar la verdad sobre la naturaleza del ser humano. Esta actitud ha venido afectando a muchos analistas, dubitativos a la hora de dar valor a lo curativo por encima de lo exploratorio y ha afectado a la delineación entre psicoanálisis en su versión tradicional y las diversas psicoterapias derivadas del mismo. Kächele aborda este tema directamente (2010) y concluye que las fronteras son muy borrosas y que carece de sentido establecer límites arbitrarios. En otro trabajo colaborativo posterior (Seybert et al, 2011) llega a plantear que hay más similitudes que diferencias entre psicoanálisis, psicoterapia de larga duración e incluso psicoterapia psicoanalítica de tiempo limitado.

Parece evidente que son los problemas y la estructura de los pacientes los que han de determinar cuál de las técnicas psicoanalíticas es la más apropiada, teniendo muy en cuenta, lógicamente, las evidencias empíricas disponibles.

\section{El psicoanálisis que se practica hoy}

La abrumadora mayoría de los pacientes atendidos por psicoanalistas, tanto en nuestro país como en cualquier otro lugar del mundo, son vistos en formatos ajenos al tradicional psicoanalítico. El grueso de los tratamientos se realizan cara a cara y con frecuencias de una o dos veces por semana. Las técnicas empleadas corresponden más a las llamadas psicoterapias psicoanalíticas, muy apropiadas para trabajar con un amplio abanico de pacientes, incluyendo a los más graves, como aquellos con organizaciones limítrofes de personalidad. Son muy habituales también los abordajes de apoyo, en ocasiones siguiendo un modelo definido (por ejemplo el de Rockland 1989) y con frecuencia constituyendo un abordaje inspirado por principios psicoanalíticos sin seguir formalmente ninguna propuesta manualizada.

Es un hecho constatable en casi todos los países que el formato tradicional de diván y múltiples sesiones semanales se ha convertido en un tratamiento para candidatos y profesionales de salud mental que desean profundizar en su conocimiento personal. Es muy improbable que la evolución de la sociedad tanto en su faceta cultural como económica permita un crecimiento relevante del número de pacientes en análisis tradicional. Schachter y Kächele (2017) señalan que los 3400 analistas de la APsA norteamericana atendían a 6000 pacientes en análisis; !una media menor de 2 !. 
Sintetizando, podemos afirmar que el grueso del trabajo de las analistas se dedica a intervenciones ajenas al formato tradicional.

\section{El programa docente en psicoanálisis}

En buena parte de los institutos que desarrollan formación la actividad docente se centra en la práctica psicoanalítica tradicional. Este modo de actuar, considerado "suicida" por autores como Kernberg (2012) supone enseñar a los profesionales en formación precisamente aquella técnica que no van a empleary a la vez no transmitir conocimientos sobre las técnicas que si van a utilizar con sus pacientes. El riesgo evidente es que un analista joven se encuentre mal preparado para emplear las técnicas que va a usar a diario al final de su entrenamiento.

El motivo de esta situación claramente anómala y casi universal tiene que ver posiblemente con la identidad de los analistas. De manera paradójica, nos aporta identidad lo que enseñamos y no lo que practicamos. Nuestra disciplina se sostiene sobre una base de creencias en algunos casos arbitrarias (Schachter \& Kächele 2012). Algunas demostradas por la evidencia acumulada desde distintos campos, otras plausibles y a la espera de demostración y otras que chocan con los datos de que disponemos y que muchos se niegan a cuestionar. Esa arbitrariedad genera una mayor necesidad de construir una identidad exigente que requiere actitudes cercanas a la fe religiosa para su sostenimiento y que implica huir de cuestionamientos y críticas que podrían poner en peligro esas creencias arbitrarias. La refutación de alguno de los planteamientos, aunque obviamente haría avanzar nuestro conocimiento pondría en serio peligro nuestra identidad, ligada a esas creencias. Y ello sería demasiado doloroso.

Algunos colegas distinguen entre psicoanalistas "sensu estricto" y profesionales que "hacen psicoanálisis". Para algunos esa es una diferencia más que relevante. Posiblemente ese precisamente es el camino, convertirnos en profesionales que "hacen" psicoanálisis y no lo convierten en el único eje de su identidad. Ello permite una mirada mucho más libre pues nuestro narcisismo no está ya ligado a una técnica o a una teoría concreta sino al propósito terapéutico o investigador que nos otorga sentido profesional. De ese modo, si nuevos conocimientos nos llevan abandonar propuestas antiguas, lo haremos sin que nuestra identidad sufra mella. Una identidad psicoanalítica firme debiera conservar una mirada siempre escrutadora y crítica, tanto de la realidad que nos rodea como de las herramientas que usamos para examinarla. 
Podría darse la paradoja de que el desarrollo auténtico del psicoanálisis precise la desaparición o el debilitamiento de la identidad como psicoanalistas. Salvo que consideremos que una verdadera identidad psicoanalítica conlleva la actitud cuestionadora y libre de la primera época, cuando algunos colegas se plantearon rechazar tópicos imperantes y prestar atención al inconsciente, al trauma, a la sexualidad infantil, a las pulsiones etc, etc. Puede que perder identidad psicoanalítica implique recuperar una identidad psicoanalítica más verdadera. No hay psicoanálisis sin cuestionamiento, empezando por el propio. La actitud de las sociedades psicoanalíticas ante la investigación es un perfecto ejemplo de la actitud del paciente que pone todo tipo de obstáculos a la libre exploración de su inconsciente. Si no se mira, todo puede seguir igual.

Un problema de compleja solución se refiere al "modelo" de psicoanálisis que se enseña a los alumnos. ¿Debe un instituto focalizarse en la psicología del yo y dejar a un lado otras aportaciones claves de la teoría psicoanalítica?. ¿Se debe en cambio transmitir una visión kleiniana si esa es la opción personal de los didactas?. ¿O una relacionalista?. ¿Es posible una enseñanza "ecléctica"? La realidad es que a día de hoy no disponemos de criterios objetivos que nos permitan comparar propuestas teóricas y desechar en su caso alguna de ellas. La opción habitual es que la docencia del Instituto se centra en aquellos autores considerados más o menos centrales prestando poca atención a los demás, especialmente los más contemporáneos. Es la tradición o el carisma lo que determina a menudo las propuestas que se enseñan y se difunden. A la espera de modos más legítimos de decidir entre propuestas a veces contradictorias, parece sensato tomar una posición "orquestal" en la transmisión de los diferentes modelos. Un Director de Orquesta debe conocer con cierta profundidad los diferentes instrumentos de la orquesta, las posibilidades de los metales, de las maderas, de la percusión, de las cuerdas... y a la vez debe ser un intérprete de primer nivel en alguno de ellos. No es posible interpretar una partitura compleja conociendo solo un instrumento ni tampoco si no se es competente en alguno. Cada equipo docente debe escoger su instrumento principal, su propuesta teórica fundamental, proporcionando además una visión realista de las características principales de otros modelos relevantes. El alumno a lo largo de su recorrido habrá de ir acercándose al instrumento que mejor se adapte a su personalidad, sus conocimientos, su experiencia clínica...y a las evidencias que vaya encontrando en su camino.

Una manera de abordar esas características principales de los diferentes modelos de modo sistemático, especialmente en su faceta clínica, puede ser partir de la propuesta de Kernberg que considera cuatro elementos centrales de la práctica psicoanalítica: la transferencia y su manejo, la interpretación y el proceso que lleva a ella, la neutralidad del analista y las condiciones para abandonarla y, finalmente, la contratransferencia y su papel 
en la cura. Parece posible examinar cada modelo clínico desde estos cuatro puntos de vista de modo sistemático, orientando así a los profesionales en formación y estableciendo criterios de comparación.

\section{Los alumnos en psicoanálisis}

La doble vertiente de nuestra disciplina antes señalada marca el tipo de alumnos a los que dirigir la enseñanza. El psicoanálisis es antes que nada una herramienta terapéutica y por ello los alumnos que aprenden a utilizar esta poderosa herramienta en un contexto sanitario han de ser clínicos, profesionales con experiencia en salud mental, en evaluación, diagnóstico y tratamiento. Se trata de una superespecialización y no de un camino alternativo. No parece adecuado que se inicie en nuestro campo con el propósito de actuar como terapeuta alguien desprovisto de ese bagaje de partida.

Por otro lado, el papel del Psicoanálisis como teoría que va más allá de la clínica y la terapia y permite explorar el mundo y sus habitantes, incluyendo los fenómenos sociales, el desarrollo normal, el arte y la creación en todas sus variantes, hace posible que personas ajenas al mundo clínico se inicien y profundicen también en el conocimiento de la teoría, aunque no la práctica, del psicoanálisis

\section{Las instituciones docentes en psicoanálisis}

Los Institutos de Psicoanálisis. Esta situación es sorprendente pues se trata de la única disciplina entre las ciencias humanas y naturales cuya enseñanza es básicamente ajena a los centros educativos y las universidades. Son agrupaciones privadas que básicamente se otorgan a si mismas la capacidad para acreditar profesionales. Este fenómeno, extendido universalmente implica que la docencia se mantiene ajena por completo a los avances y a los cambios de la educación y, sobre todo, ajena a la investigación y al influjo de otros campos del saber.

Es habitual que los institutos no consideren apropiado formar a sus estudiantes en disciplinas vecinas, ni siquiera en aportaciones teóricas de otros modelos psicoterapéuticos. Se presenta el psicoanálisis como algo cerrado y completo, sin contacto con otras ciencias, ni siquiera con las más cercanas de la medicina o la psicología clínica. Se plantea la fantasía de que un analista no requiere ningún conocimiento fuera de nuestro campo para convertirse en un terapeuta de primer nivel. 
Parece imprescindible para el futuro del psicoanálisis su entrada, o su retorno, a la Universidad, lugar de donde para algunos de nosotros nunca debiera haber salido. Y no se trata solo de la inclusión de analistas en los departamentos de psiquiatría o psicología, sino de que la formación y acreditación de nuevos profesionales se haga desde la propia Universidad. Sin duda no es práctico y quizá no es necesario que los Institutos pierdan su papel actual pero parece imprescindible que se desarrollen centros universitarios dedicados a la formación, acreditación y, sobre todo investigación en y sobre psicoanálisis.

Ciertamente, la Universidad acarrea sus propios problemas, entre ellos una importante falta de recursos y una burocracia gigantesca. Las Facultades de Medicina en España, sin ir más lejos, se enfrentan a un verdadero riesgo de desaparición, por la peligrosa confluencia de falta de financiación, de profesores, de clínicos e incluso de médicos, en sus estructuras. Pero reconociendo todo esto, parece evidente que es la Universidad donde deben ser transmitidos los saberes y que es responsabilidad de docentes, Gobierno y la Sociedad en su conjunto, transformar esa institución en el lugar de creación y transmisión de conocimientos que debiera ser.

También debe enseñarse teoría psicoanalítica fuera de los lugares donde se forman psicoanalistas. Nuestra disciplina debería tener un lugar en las enseñanzas de grado y posgrado de las Facultades de Medicina o Psicología y en los programas formativos de residentes de Psicología Clínica y Psiquiatría. También sin duda puede ser parte integrante de los programas docentes dirigidos a trabajadores sociales, enfermeras y otros profesionales sanitarios. De nuevo, es responsabilidad de quienes practicamos psicoanálisis esta labor didáctica asertiva, saliendo al encuentro de quienes podrían estar interesados en nuestra disciplina y beneficiarse de ella. Una labor de tono quizá apostólico muy necesaria.

\section{Metodología de enseñanza en Psicoanálisis}

El entrenamiento psicoanalítico continúa cien años después basándose en tres pilares fundamentales: los seminarios de teoría, el análisis personal o "didáctico" y las supervisiones de casos. Seminarios y supervisiones constituyen las herramientas docentes habituales en la transmisión de todos los saberes técnicos que implican un "hacer". Un cirujano asiste a presentaciones en las que alguien muestra en detalle una técnica quirúrgica explicando su base teórica, detallando la intervención, sus dificultades, complicaciones.... Luego el estudiante observa al maestro enfrentarse a los problemas que ha descrito a través de su presencia directa o indirecta en el quirófano. Finalmente, el estudiante lleva a cabo personalmente las intervenciones bajo la mirada atenta del colega 
más experto, hasta que se considera que ha adquirido suficiente soltura con el procedimiento

Solo en Psicoanálisis se plantea como obligación que el estudiante pase por la experiencia que van a vivir los pacientes. Sin duda este paso implica elementos importantes y que debemos valorar junto a otra característica muy propia de nuestra disciplina. Habitualmente no vemos trabajar a los docentes, salvo con nosotros durante nuestro análisis "didáctico". Nuestro análisis se convierte así no solo en una oportunidad para explorar nuestro mundo interno y solucionar alguno de nuestros conflictos sino también en la única experiencia directa del trabajo de un experto. Por ello no es extraño que a menudo los analistas pasen por más de una experiencia de análisis personal. No sólo por la conveniencia de trabajar aspectos insuficientemente abordados en esas terapias "didácticas" sino también por la necesidad, más o menos consciente, de ver y sentir como trabaja un colega experto. Podemos imaginar a un cirujano que se apresta a llevar a cabo operaciones complejas con una técnica nueva y nunca ha visto desarrollarla a quienes la conocen bien, sino que se limita a ser supervisado ocasionalmente mientras desarrolla su trabajo.

El propio término "candidato", ajeno a cualquier institución pedagógica, revela aspectos importantes. A los formandos en un centro educativo, les denominamos habitualmente de un modo que describe su tarea: estudiantes, por ejemplo, o profesionales en formación, o alumnos. El término candidato implica una posición de espera a ser elegido por otros y ya conlleva implícita la actitud de resultar atractivo y a la vez gratificar las expectativas y deseos de quienes han de escogernos.

En la jerarquía de las sociedades psicoanalíticas el analista didacta ocupa el lugar superior de la pirámide. Se trata de profesionales senior, con años de experiencia clínica y que son escogidos por un comité didáctico para ese cargo. La condición de didactas implica un reconocimiento de la capacidad de enseñar teoría, de supervisar el trabajo de los candidatos $y$, sobre todo, de realizar análisis didácticos. Este esquema implica que esas personas reúnen capacidades muy distintas, a veces muy alejadas unas de otras. Un clínico excelente no tiene por qué ser un buen supervisor y tampoco un docente brillante. En un panorama como el actual, en el que los pacientes en análisis tradicional son escasos más allá de los candidatos, se da la situación de que solo los didactas tienen acceso frecuente a tratamientos tradicionales, mientras que los otros analistas se ven privados de esa importante fuente de casos. Las consecuencias de esta situación en términos de poder institucional y rendimientos económicos son evidentes. El hecho de que un candidato solo pueda optar a ser analizado por un didacta del propio Instituto, práctica habitual, mezcla 
de modo inadecuado aspectos terapéuticos con formativos y burocráticos y propicia sin duda que el profesional en formación deje fuera de su análisis aquellos aspectos que intuye pudieran resultar menos atractivos para el didacta que le analiza y que, también, representa a la institución y a quienes han de escoger al candidato en el futuro.

Muchos autores han abordado este complejo problema (por ej. Schachter \& Kächele 2017, Kernberg, 2019, 2016, Kernberg \& Michels 2016, Auchincloss \& Michels 2003, Wallerstein 2010...) y cada vez hay más apoyos a la idea de que el análisis personal de los alumnos debe quedar completamente aislado del recorrido docente. Se plantea además que los profesionales en formación puedan, o incluso deban, buscar su analista fuera de su instituto, con lo que la independencia puede quedar asegurada. De esta manera , las posibilidades de que el formando deje fuera de su proceso analítico aspectos menos atractivos para sus maestros no desaparece pero si se reduce. Es evidente sin embargo que un cambio de estas características puede despertar resistencias importantes al tener consecuencias no solo educativas, sino organizativas o incluso económicas en las sociedades.

\section{La investigación en Psicoanálisis}

Para Horst Kächele y otros muchos autores (Schachter \& Kächele 2017, Leuzinger, Solms \& Arnold 2020, Kernberg 2006, 2015, Fonagy 2013, Safran 2012, etc.) el futuro del psicoanálisis pasa por la investigación. Tanto por el desarrollo de estudios como por la difusión de los resultados. Muchos tenemos la impresión de que la mayoría de los psicoanalistas desconocen los abundantes resultados ya disponibles en torno a la eficacia de las intervenciones psicoanalíticas en todas sus formas. La actitud habitual es bien de arrogante indiferencia hacia la investigación o de simple ignorancia de la misma. La consecuencia de esto es que gran cantidad de evidencias que apoyan nuestras teorías, nuestras propuestas teóricas y nuestra actividad clínica no son conocidas ni comunicadas. $Y$ esta es una responsabilidad absoluta de los que practicamos psicoanálisis pues no es esperable que colegas que se dedican a otras actividades sean quienes busquen y difundan estudios ajenos a su quehacer. El futuro del psicoanálisis pasa por la atención a la investigación. Si queremos mantener una presencia importante en la sociedad y en los sistemas sanitarios es imprescindible profundizar en nuestros conocimientos modificando aquellas prácticas que se demuestran inadecuadas, adoptando nuevas maneras de actuar refrendadas por los estudios y manteniendo una formación continuada de los profesionales difundiendo los nuevos hallazgos, positivos y negativos, dentro y fuera de la comunidad psicoanalítica. 
Existe un número relevante de compilaciones de trabajos de investigación en nuestra disciplina (por ej. Poch \& Avila 1997, Fonagy et al. 2002, Levy et al 2012,) y multitud de recomendaciones sobre los problemas metodológicos a resolver (Por ej. Kächele. 2012, Kächele \& Erhardt 2012, Kächele 2013, Schachter \& Kächele 2010, 2013, Kächele \& Schachter 2014, Taubner et al 2012) Las líneas maestras de lo que debiera ser la investigación del futuro en psicoanálisis están ya demarcadas. Podemos citar aquí algunas

1. Acuerdo básico en terminología y precisión conceptual. Es imprescindible contar con un "catálogo" de conceptos fundamentales que sean definidos nítidamente. Sería ingenuo esperar que un colectivo tan heterogéneo como el psicoanalítico se pusiera de acuerdo para llevar a cabo esta obra magna. Pero si sería factible que un grupo de investigadores de prestigio en nuestro campo planteara un catálogo de ese tipo, que luego pudiera servir de referencia para el resto de investigadores. Algunos analistas ligados al comité de investigación de la IPA y el programa RTP (Research Training in Psychoanalysis) de esa institución han llevado a cabo esfuerzos en ese sentido. Tendría sentido que, siguiendo la tradición psicoanalítica, se empiece por precisar conceptos estrechamente ligados a la clínica, pasando posteriormente a elementos de la metapsicología más alejados de la actividad terapéutica.

2. Acuerdo básico en diagnósticos y métodos de evaluación. Aunque a algunos les suene a anatema, es vital contar con un "DSM" psicoanalítico, un sistema diagnóstico que recoja los elementos fundamentales de la evaluación psicoanalítica, y nos permita operacionalizar en cierta medida las valoraciones estructurales o de organización de personalidad. Esfuerzos valiosos como el Psychodynamic Diagnostic Manual, de Lingiardi \& MacWilliams (2017) o Instrumentos como la STIPO (Clarkin et al 2007) constituyen un punto de partida.

3. Colaboración. Los tratamientos psicoanalíticos son de larga duración y requieren muchas horas de trabajo cada uno. Ello conlleva una enorme dificultad para alcanzar tamaños muestrales relevantes en los estudios, lo que a su vez dificulta encontrar significación estadística a las diferencias halladas en los estudios. La consecuencia de todo esto es la imperiosa necesidad de colaboración entre instituciones y profesionales. Los estudios de investigación en psicoterapia y en psicoanálisis, más complejos en su diseño y más difíciles de implementar que los estudios de investigación psicofarmacológica, requieren que analistas e instituciones diversas compartan el estudio y su desarrollo. Ciertamente la división actual de nuestro colectivo en mil y una sociedades con escasa relación no favorece este requisito central. Pero se trata de una condición sine qua non. Sin colaboración, no hay investigación y no hay futuro. 
4. Financiación. Los estudios en y sobre psicoanálisis no cuentan con el apoyo de ninguna industria que pueda beneficiarse de los resultados obtenidos. Por otro lado, la mayoría de las sociedades psicoanalíticas no disponen de recursos suficientes y no dedican parte de ellos a la investigación. Solo algunas grandes agrupaciones de sociedades, como la IPA, ha dedicado una cierta atención a la investigación propiciada por la influencia de algunas figuras de nuestra profesión. Pero esa atención ha sido a menudo dubitativa y proyectos que antes poseían gran fuerza se ven ahora arrinconados, tanto por una política de restricción de gastos como por un menor interés hacia la investigación. Sólo queda la posibilidad de obtener financiación de las instituciones públicas o privadas dedicadas a esas actividades. Y para ello es imprescindible contar con proyectos metodológicamente bien presentados y que puedan competir con otros proyectos ajenos a nuestro campo que pugnan también por una parte de los recursos, siempre limitados. La presencia en la Universidad es también clave en este aspecto pues facilita mejoras evidentes en el diseño de los proyectos y en el acceso a fondos.

5. Formación en investigación. Los conocimientos sobre investigación en Psicoanálisis debieran ser parte del curriculum formativo de todos los institutos. Esto evidentemente implica incluir investigadores entre los didactas. Los pasos a dar son varios.

A) Familiarizar a los "candidatos" y a toda la comunidad con la relativamente extensa bibliografía sobre investigación. Esta es en buena parte desconocida por los propios psicoanalistas y por tanto no se difunde en absoluto fuera de círculos muy pequeños.

B) Formación en lectura crítica. Tanto las publicaciones de investigación conceptual o las reflexiones clínicas tradicionales en nuestro campo, como la investigación empírica, requieren lecturas alejadas de la exégesis y dirigidas a la valoración crítica y rigurosa de los argumentos presentados y la metodología propuesta. Esta lectura crítica no suele formar parte del entrenamiento en los institutos y es vital para conseguir nuevas generaciones que vean el examen de publicaciones como una plataforma desde la que mejorar la práctica clínica y avanzar en la teoría y no como una salmodia meditacional.

C) Formación básica en metodología de investigación. Fundamentos de la reflexión clínica en psicoanálisis. Fundamentos de la investigación conceptual. Estudios de caso único. Metodología básica de investigación empírica. A menudo los clínicos se ven abrumados por las técnicas de análisis estadístico que se suelen utilizar en investigación empírica. El temor es infundado. La estadística habitualmente utilizada es más fácil de evaluar de lo que parece y a menudo el análisis estadístico se limita solo a comprobar hasta qué punto los resultados hallados pueden ser debidos al azar. Es la metodología la clave de 
cualquier estudio y lo que determina su valor; también en nuestro campo. Los seminarios sobre epistemología, que sí aparecen en los programas de algunos institutos, podrían casar bien con la enseñanza de metodología y lectura crítica. De nuevo Kächele puede ser aquí un guía excelente (Buchholz \& Kächele 2018).

D) Formación avanzada. Sería lógico a partir de ahí que algunos estudiantes pudieran adquirir una formación más sólida de modo que fueran integrándose en los equipos dedicados a la investigación. Sería ideal la aparición en todos los institutos de investigadores-clínicos que combinaran ambas dedicaciones y sirvieran de puente para trasladar los conocimientos de la investigación al resto del Instituto y a la Comunidad científica. Parece lógico que esos miembros con mayor dedicación a la investigación estén vinculados también a la Universidad

\section{Papel social del Psicoanálisis}

Otra responsabilidad más de los psicoanalistas es poner nuestros conocimientos al servicio de la comunidad. Y esto pasa por una acción decidida para hacer llegar a toda la población, y no solo a la más pudiente, los tratamientos que desarrollamos. Son dos las vías principales para ello. Por un lado incorporar tratamientos psicoanalíticos a la oferta sanitaria pública. Hay abordajes de tiempo limitado de gran eficacia, hay abordajes de apoyo que pueden sustituir con ventaja o complementar a otras intervenciones, hay por fin psicoterapias psicoanalíticas de larga duración que pueden aportar posibilidades de mejoría extraordinarias a pacientes de gran severidad, evitando hospitalizaciones, tentativas suicidas y graves deterioros de la vida personal. Los abordajes grupales, tanto aquellos de sólido basamento psicoanalítico (intervenciones grupoanalíticas, por ej.) como aquellos otros formatos que contienen elementos psicodinámicos, son también ejemplo de técnicas que deben formar parte de la oferta sanitaria pública. Todo ello sin hablar de dotar a los clínicos de una forma de evaluar los casos y una manera de observar y escuchar de ese modo personalizado, profundo y compasivo que caracteriza al psicoanálisis. Otra vía complementaria es ofertar a los ciudadanos la posibilidad de recibir tratamientos en las sociedades e institutos de forma gratuita o con costes reducidos. Algo que en general sucede con poca frecuencia y que podría significar una contribución importante de nuestras agrupaciones a los ciudadanos que sufren.

Obviamente la teoría psicoanalítica permite reflexionar sobre la conducta de individuos y grupos fuera de la clínica y desde luego supone una ventana desde la que observar los productos culturales y la actividad creativa. Pero no deberíamos olvidar que es la clínica el terreno fundamental, en el que el verdadero valor del psicoanálisis se ha de poner de 
manifiesto. El objetivo de nuestra intervención sigue siendo, cien años después, el mismo: ayudar al paciente a avanzar en sus relaciones afectivas, en su trabajo y actividad productiva y en su capacidad de disfrute y creatividad. No se trata de un mero ejercicio intelectual apasionante para analista y analizando sino una intervención terapéutica destinada a cambiar profundamente la estructura interna del paciente y con ello reducir su sufrimiento y posibilitarle una vida más plena. En otras palabras, la meta del psicoanálisis es la cura.

Podemos terminar citando una vez más a Horst Kächele (2010): "Desde el diverso y heterogéneo caleidoscopio de las prácticas y teorías psicoanalíticas, emerge una conclusión con razonable certeza. Todos los terapeutas psicoanalíticos deben aproximarse a su tarea con una profunda sensación de humildad. Las convicciones de base endeble acerca de una visión analítica particular pueden impedir la monumental evaluación empírica que se extiende ante nosotros".

\section{REFERENCIAS}

Auchincloss, E.L. and Michels, R. (2003). A reassessment of Psychoanalytic education. Int. J. Psycho-Anal., 84(2):387-403

Buchholz, M.B. Kächele, H. (2018). Teaching research methods to psychoanalysts: Experiences from PSAID. Int. Forum Psychoanal., 27(2):114-120.

Clarkin, J., Caligor, E., Stern, B., Kernberg, O. (2007). Structured Interview of Personality Organizations (STIPO). Personality Disorders Institute. Weill Medical College of Cornell University

Fonagy, P. Ed. (2002). An open door review of outcome studies in psychoanalysis. Second revised edition. London: International Psychoanalytical Association

Fonagy, P. (2013). There is Room for Even More Doublethink: The Perilous Status of Psychoanalytic Research. Psychoanal. Dial., 23(1):116-122

Freud S (1981). [1933]. Nuevas lecciones introductorias al psicoanálisis. En Obras Completas. Tomo III. Pp 3101-3206. Madrid: Biblioteca Nueva

Kächele, H. (2010). Distinguishing Psychoanalysis from Psychotherapy. Int. J. Psycho-Anal., 91(1):35-43.

Kächele, H. Erhardt, I. (2012). Is it Possible to Measure Countertransference?. Rom. J. Psychoanal., 5(1):91-105.

Kächele, H. (2013). Manualization as Tool in Psychodynamic Psychotherapy Research and Clinical Practice-Commentary on Six Studies. Psychoanal. Inq., 33(6):626-630.

Kächele, H. Schachter, J. (2014). On Side Effects, Destructive Processes, and Negative Outcomes in Psychoanalytic Therapies: Why Is It Difficult for Psychoanalysts to Acknowledge and 
Address Treatment Failures?. Contemp. Psychoanal., 50(1-2):233-258.

Kandel, E. (1999). Biology and the future of Psychoanalysis: a new intellectual framework for psychiatry revisited. Am J Psychiatry, 156, 505-24.

Kernberg, O.F. (2006). The Pressing Need to Increase Research in and on Psychoanalysis. Int. J. Psycho-Anal., 87(4):919-926

Kernberg, O.F. (2012). Suicide Prevention for Psychoanalytic Institutes and Societies. J. Amer.Psychoanal. Assn., 6o(4):707-719.

Kernberg, O.F. (2015). Resistances and Progress in Developing a Research Framework in Psychoanalytic Institutes.Psychoanal. Inq., 35S(Supplement):98-114

Kernberg, O.F. (2016). A Proposal for Innovation in Psychoanalytic Education. DIVISION/Rev., 15:13-16

Kernberg, O.F. and Michels, R.(2016). Thoughts on the Present and Future of Psychoanalytic Education. J. Amer. Psychoanal. Assn., 64(3):477-493

Kernberg, O. (2019). Treinta métodos para destruir la creatividad de los candidatos a psicoanalistas. Rev. Psicoanál. Asoc. Psico. Madrid, 85:47-62

Lingiardi V, McWilliams N. (2017). Psychodynamic Diagnostic Manual. Second Edition. New York: The Guilford Press.

Leuzinger-Bohleber, M., Solms, M., and Arnold, S.E. Eds. (2020). Outcome Research and the Future of Psychoanalysis. Clinicians and Researchers in Dialogue. London: Routledge

Levy, R.A., Ablon, J.S., Kächele, H. Eds. (2012). Psychodynamic Psychotherapy Research: Evidence-Based Practice and Practice-Based Evidence. Humana Press.

Poch J, Ávila Espada A. (1997). Investigación en Psicoterapia: La contribución psicoanalítica. Barcelona: Paidós.

Rockland, LH. (1989). Supportive Therapy: A Psychodynamic Approach. New York: Basic Books.

Roth A, Fonagy P, Parry G, Target M, Woods R. What works for whom? A critical review of Psychotherapy research. 2nd ed. 2004.

Safran, J.D. (2012). Doublethinking or Dialectical Thinking: A Critical Appreciation of Hoffman's "Doublethinking" Critique. Psychoanal. Dial., 22(6):710-720

Schachter, J. Kächele, H. (2010). The Couch in Psychoanalysis. Contemp. Psychoanal., 46(3):439459.

Schachter, J. and Kächele, H. (2013). An Alternative Conception of Termination and Follow-up. Psychoanal. Rev., 100(3):423-452

Schachter, J. Kächele, H. (2012). Arbitrariness, Psychoanalytic Identity and Psychoanalytic Research. Rom. J. Psychoanal., 5(2):137-161.

Schachter, J; Kächele, H. (2017). Nodal Points: Critical Issues in Contemporary Psychoanalytic Therapy. International Psychoanalytic Books. 
Seybert, C., Desmet, M., Erhardt, I., Hörz, S., Mertens, W., Ablon, J.S., Levy, R. and Kächele, H. (2011). Therapists' Techniques in Psychoanalysis and Short-Term and Long-Term Psychoanalytic Psychotherapy: Are They Different?. J. Amer. Psychoanal. Assn., 59(3):592-602

Solms, M. (2020). The scientific basis of Psychoanalysis. Chapter 1. En Outcome Research and the future of Psychoanalysis. Leuzinger-Bohleber, M; Solms, M; Arnold, E. Eds. London \& New York. Routledge

Stoller, RJ. (2009). Sweet Dreams. Erotic plots. London: Karnac Books.

Taubner, S. Buchheim, A. Rudyk, R. Kächele, H. Bruns, G. (2012). How Does Neurobiological Research Influence Psychoanalytic Treatments?-Clinical Observations and Reflections from a Study on the Interface of Clinical Psychoanalysis and Neuroscience. Am. J. Psychoanal., 72(3):269-286.

Thomä, H. y Kächele, H. (1989). Teoría y Práctica del Psicoanálisis. Vol. 1. Fundamentos. Barcelona: Herder [original de 1985].

Thomä, H. y Kächele, H. (1990). Teoría y Práctica del Psicoanálisis. Vol. 2. Estudios Clínicos. Barcelona: Herder [original de 1987].

Wallerstein, R. S. (2010). The training analysis: Psychoanalysis' perennial problem. Psychoanalytic Review, 97, 903-936.

Original recibido con fecha: $\quad 30 / 9 / 2020 \quad$ Revisado: 30/9/2020 Aceptado: 30/09/2020 